\title{
Bioimpedance Measurement in the Kidney Disease Patient
}

\author{
Joëlle Cridlig, Mustapha Nadi and Michèle Kessler \\ Université Henri Poincaré Nancy 1
}

France

\section{Introduction}

\subsection{The problematic of hydration status in the kidney disease patient}

Dry weight corresponds to the body weight of a person with normal extracellular fluid volume [1]. In the context of hemodialysis, dry weight is the weight reached the end of the dialysis session by patients who will remain free of orthostatic hypotension or hypertension until the next session. Clinicians are thus obliged to estimate the appropriate dry weight each individual patient should reach at the end of a dialysis session. If this weight is underestimated, the patients are at risk of various incidents ranging from simple yawning to death. Low dry weight also carries a permanent risk of hypotension, cramps, nausea, vomiting or ischemia. If this weight is overestimated, chronic hyperhydration can cause acute events including pulmonary edema, or hypertension, but also long-term consequences affecting cardiovascular morbidity and mortality [2]. This important notion of dry weight is however quite problematic because it corresponds to a transient state, making it necessary to anticipate weight gain between two dialysis sessions and thus to reach a certain degree of dehydration at the end of each hemodialysis session.

The many methods proposed for measuring dry weight and body composition are still under investigation. The difficulty encountered in establishing a reliable comparative tool for measuring these parameters arises from inevitable physiological, anatomical and physical variability. Most approaches remain empirical, relying on feedback from trial and error [3].

In practice, dry weight is estimated clinically [4]. Physical examination is a classic but limited tool. Solid evidence-based analysis of specific physical findings such as systolic blood pressure, orthostatic blood pressure, or the presence or not of edema is lacking. Such clinical symptoms can also be related to conditions other than a dry weight or body composition problem. Several tests have been proposed to assess the dry weight of hemodialysis patients [5]. Echocardiographic inferior vena cava diameter and biochemical markers are available but results exhibit high variability and poor correlation with extracellular volume; there are also unserviceable in detecting volume depletion.

Bioimpedance spectroscopy measurement has been demonstrated as a potentially useful method to determine the physiological status of living tissues [6]. Disease-related alterations are associated with variations in essential tissue parameters such as physical structure or ionic composition that can be detected as changes in passive electrical properties. 
Bioimpedance spectroscopy can be used as a diagnostic tool reflecting the overall status of a patient or of an individual organ. The range of applications derived from this method is wide making this non-destructive and non-invasive approach a promising technique for the characterization of the physiological status of the human body.

This chapter presents the use of bioimpedance spectroscopy as a tool for measuring the dry weight of the hemodialysis patient. The first part summarizes the basic fundamentals of the method, describing the underlying electrical and biological principles and the potential interest for applications in hemodialysis. Techniques and models are presented and discussed. The second part discusses clinical results obtained at the hemodialysis center of the Nancy University Hospital, with focus on patient-related parameters influencing or limiting measurements and their interpretation.

\subsection{Basic principles of bioimpedance spectroscopy}

\subsubsection{Physical principles}

The bioelectrical properties of the human body depend on the nature of the biological tissues composing it and their relative conductivities. These properties lead to the notion of impedance of a biological medium which varies as a function of the frequency of an electrical current applied across it. This in turn leads to the notion of bioimpedance spectroscopy.

Impedance is a complex quantity $\underline{(\mathbf{Z})}$ describing, in compliance with Ohm's law (assuming sinusoidal voltage), the relationship between the voltage difference across the medium and the amplitude of the electrical current. Because of the capacitive nature of biological media, all impedance measurements of the human body involve a phase shift between the voltage and the current, yielding complex values. Biological media are weak conductors due to the dissipation (or loss) of energy in the media and can be considered as energy-loosing dielectrics. The quantities of interest can thus be defined using a schematic representation of a biological suspension placed between two electrodes (Fig. 1).

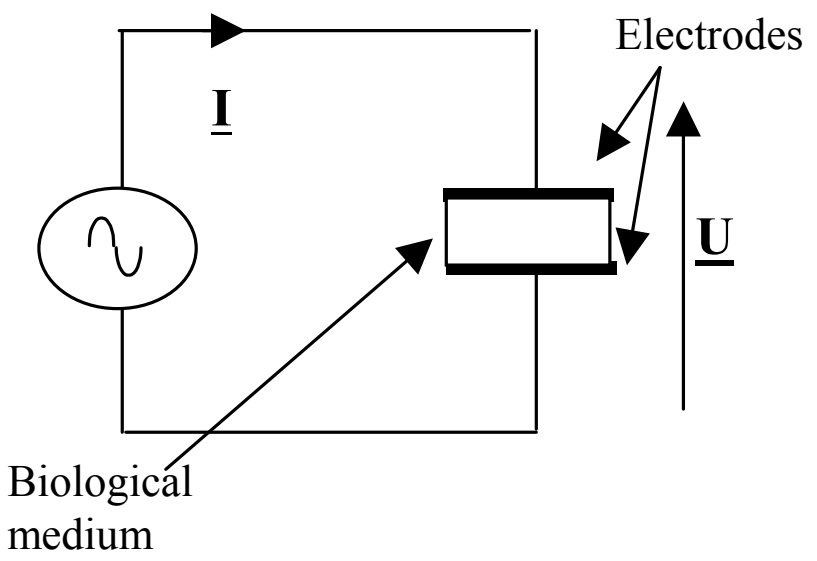

Fig. 1. Basic principle of bioimpedance spectroscopy 
$\underline{\mathbf{U}}$ and $\underline{\mathbf{I}}$ are sinusoidal quantities with phase shift $\phi$

$$
\underline{\mathrm{U}}=\mathrm{Uo} \mathrm{e}^{\mathrm{j} \omega \mathrm{t}} ; \underline{\mathbf{I}}=\operatorname{Io} \mathrm{e}^{\mathrm{j}(\omega \mathrm{t}+\phi)}
$$

The impedance of a biological medium is thus the relationship between the voltage difference across the medium and the current amplitude as described by Ohm's law:

$\underline{\mathbf{U}}=\underline{\mathbf{Z}} \underline{\mathbf{I}}$ where $\underline{\mathbf{Z}}$ is the complex impedance expressed in Ohms $(\Omega)$. In Cartesian form this gives $\underline{Z}=R+j X$ where $R$ is the resistance, $X$ is the reactance, both expressed in Ohms, and $j$ the imaginary unit, $j=\sqrt{-1}$

Setting $Z=\sqrt{R^{2}+X^{2}}$ and $\phi=\operatorname{arctg}(X / R)$ gives the polar form $\underline{Z}=Z e^{j} \phi$

The bioelectric impedance $\underline{Z}$ describes the inertia of a biological medium opposing passage of a sinusoidal current with intensity $\underline{\mathbf{I}}$ and pulsation $\omega(\mathrm{rad} / \mathrm{s})$. Along the lines of the electrical current, this impedance generates a difference of potential $\underline{\mathbf{U}}$. Bioelectrical impedance can be represented in a complex plane. It is perfectly defined by knowledge of either its real and imaginary components ( $R$ and $X)$, or by its module and its argument, $\underline{Z}$ and $\phi$.

For an isotropic, homogeneous, linear medium, impedance is a function of the medium's electrical properties, conductivity and permitivity, but also depends on the geometric features of the measurement cell. This so-called bioimpedance is a well-known tool for characterizing different physiological water compartments [7]. Because of the complexity and highly heterogeneous nature of living tissues, a global approach is used, considering the whole body as a suspension of cells in water and electrolytes [8].

\subsubsection{Biological basis}

Biological tissues cannot be considered as ideal conductors. They are ionic conductors with heterogeneous structures. If we limit our considerations to the sole electrical aspect of interest here, biological tissues can very schematically be considered as a combination of two components: $i$ ) a free water medium called the extracellular fluid, within which are suspended ii) cells surrounded by a membrane containing and thus limiting a volume of intracellular fluid (Fig. 2). The cell concentration can vary greatly, depending on the nature of the tissue.

The fluid components (extracellular fluid, plasma and intracellular fluid) can be considered as electrolytic suspensions of ions whose concentration, electrical charge and mobility, taking into account the viscosity of the medium, will essentially determine the impedance of the suspension, mainly arising from resistive type resistance. Cell membranes however constitute a more complex component. Cole demonstrated that cell membranes can be likened to capacitors $[9,10,11]$. This divides biological media into two basic components, resistors (R) and capacitors (C), which when connected in parallel produce an electrical equivalent model as described by Fricke [13] (Fig. 2). This simplified model $\left(R_{e}, R_{i}\right.$ and $C$ are not ideal) enables an interpretation of observed biophysical phenomena.

Theoretically, tissue impedance, like any electric impedance, can be measured. The mass of tissue to study simply has to be delimited and linked to a measurement device via a system of electrodes. In practice however, measuring the electrical characteristics of biological tissues raise many specific problems. It is useful to recognize the electrical properties of 
biological tissues and their components because of their interest both in medicine, where many diagnostic methods are based on electrical principles, and in fundamental physiology, where these same properties contribute to the structural analysis of cell organization, the study of cell excitation mechanisms, or to the analysis of protein molecules. Debye, ColeCole and Maxwell-Wagner models have been developed to represent the theoretical interpretations of these phenomena. These models can be used to demonstrate important factors characterizing biological tissues [12].

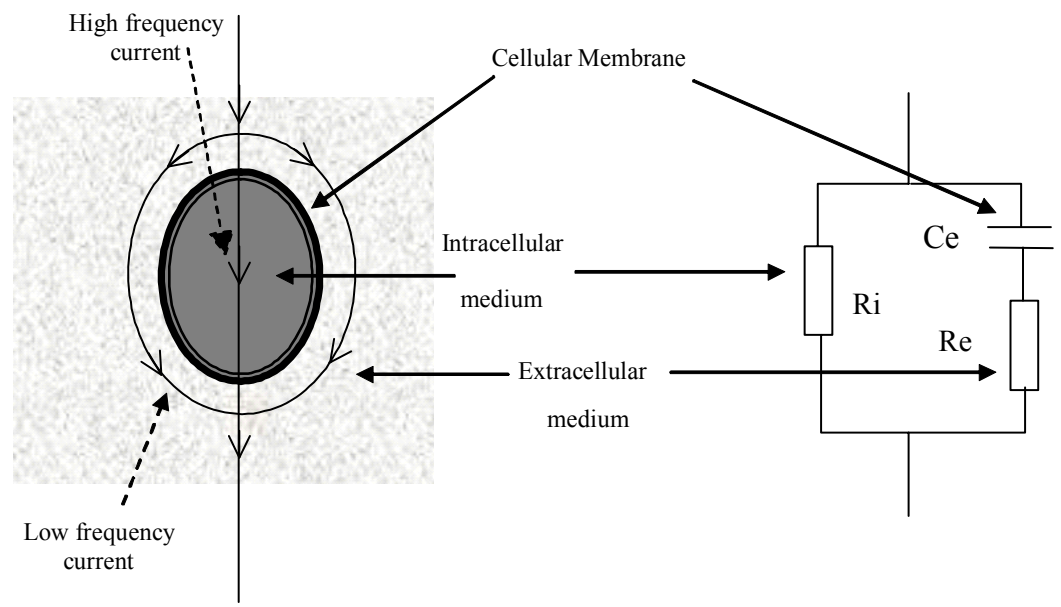

Fig. 2. Electrical equivalent model (Fricke's model) of a biological cell in suspension in the extracellular fluid. Ri : intra-cellular resistance, Re : extra-cellular resistance, Ce : capacitance

Capacitance and resistance can be combined either in series or in parallel, leading to several variants of the Fricke model. Resistivity (charge accumulation) and conductivity (conduction of electrical current) are however two independent processes for which the parallel model has been found to be more representative. Formulas used for biological tissues are thus all based on the Fricke model [13]. Applying these models to bioimpedance spectroscopy curves allows a quantification of value differences corresponding to the intracellular fluid and the extracellular fluid and thus their corresponding masses. The interest for hemodialysis is obvious.

\subsection{Usefulness of bioimpedance for the kidney disease patient}

Much research has been devoted to the determination of body hydration status and fluid compartments, particularly in hemodialysis patients [14]. Work has focused on various physiological parameters with different modalities to study individual segments of the body or the whole body $[15,16,17]$ using multiple or unique frequencies [18]. One of the goals is to improve predictive equations and to identify the effect of different parameters on the measures obtained $[19,20]$. In dialysis for example, it has been shown that fluid is removed predominantly from the extracellular and peripheral (arms, legs) compartments [21]. Thus, different biofluid models have been proposed to describe the whole body or individual organs. Basically, applying the bioimpedance method to determine body composition 
consists in placing electrodes conveniently on the arms and legs in order to impose an electrical current and measure the voltage it induces across the body. For a reasonable current density, the behavior is linear so that Ohm's law remains valid. Intra- and extracellular fluids are mainly resistive, whereas cell membranes act as an insulator between these two compartments. Rare bioimpedance spectroscopy studies conducted in kidney graft recipients have shown a trend towards improved hydration after transplantation. We have been unable to find any study evaluating hydration changes in patients with acute kidney disease.

\section{Theoretical and experimental models}

\subsection{Principles of bioimpédance in hemodialysis}

Many different instruments have been marketed using algorithms based on different equations predicting body composition from impedance measurements [22, 23, 24, 25]. The validity of these different equations remains a question of debate since most have been established empirically. Moreover, the equations actually used by the algorithms of commercial microcomputers are not readily accessible, making it rather difficult to discuss their validity or make necessary corrections. The voltage produced by the current is measured to calculate the impedance. The relationships between impedance and other variables such as body water volume have been established using statistical correlations observed in specific populations rather than on a real biophysical basis. Actually, the theoretical basis can be summarized by the following statement: the human body is a complex conductive volume composed of heterogeneous tissues and intra- and extracellular compartments in perpetual movement.

Basically, the algorithms applied are based on regression laws used as a tool predictive of the relationship between two or more body variables constituting a database. Thus for total body water (TBW) the regression equation is written as [26](with $\mathrm{H}$ : Height):

$$
\mathrm{TBW}=\mathrm{a} \cdot \mathrm{H}^{2} / \mathrm{R}+\mathrm{b} \cdot \text { weight }+\mathrm{c} \cdot \text { Age }+\mathrm{d}
$$

TBW is measured in a large population using a gold standard, e.g. isotopic dilution (Fig. 3). The statistical software then uses regression analysis to establish the best fitting equation describing the relationship between TBW and the different measurements, e.g. height, weight, age, gender, resistance... For subsequent resistance measurements, a software inserts the recorded data into the accepted formula and delivers the results as TBW (Fig. 4).

\subsubsection{Prediction of total body water}

\subsubsection{Historical background}

One of the most commonly cited relationship is the cylinder model where the volume of a conductive cylinder is function of its length (L) and its resistance (R). High frequency current penetrates into the cell and runs across body fluids. A TBW value can thus be obtained by modeling the human body as a sum of cylinders. Devices applying this method are calibrated by dilution techniques. These devices rely on the following relationship:

$$
\mathrm{TBW}=\left(\mathrm{a} \cdot \mathrm{H}^{2}\right) / \mathrm{R}+\mathrm{c}
$$




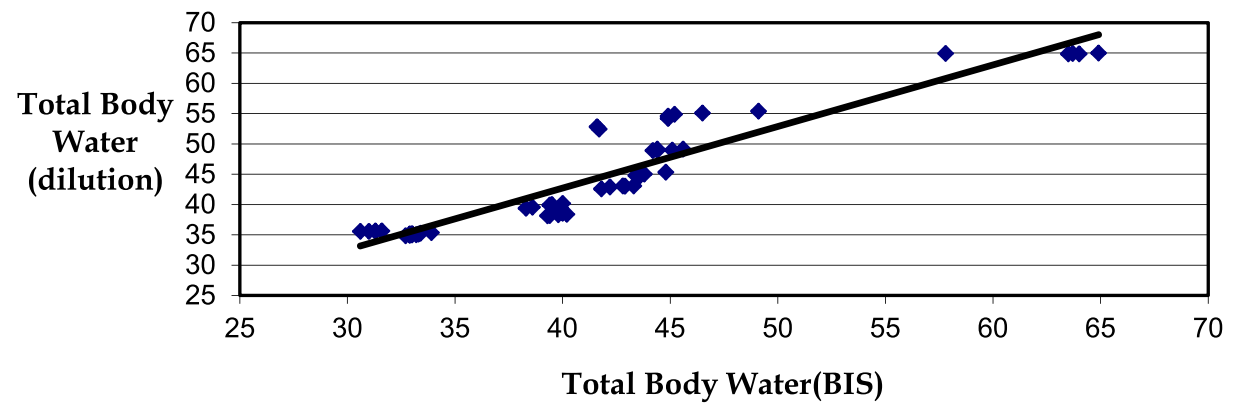

Fig. 3. Correlation between isotopic dilution and bioimpedance (BIS)

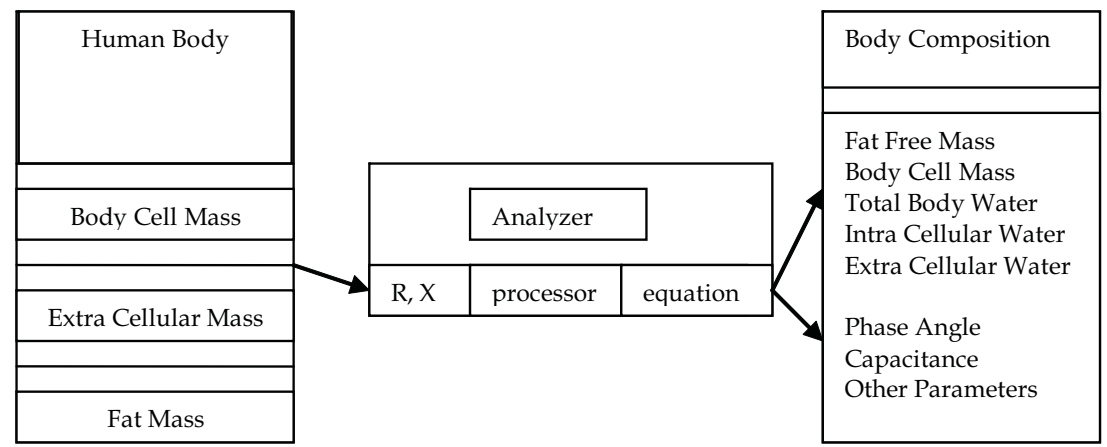

Fig. 4. Exemple of bioimpedance analysis block diagram [23] ( $R$ : resistance, $X$ : Reactance)

Thomasset $[27,28]$ was the first, in 1963 , to use two frequencies (1 et $100 \mathrm{kHz}$ ), to measure extracellular and total water respectively using the Cole-Cole model (Fig.5). Subsequently, several different formulas have been proposed based on regression equations.

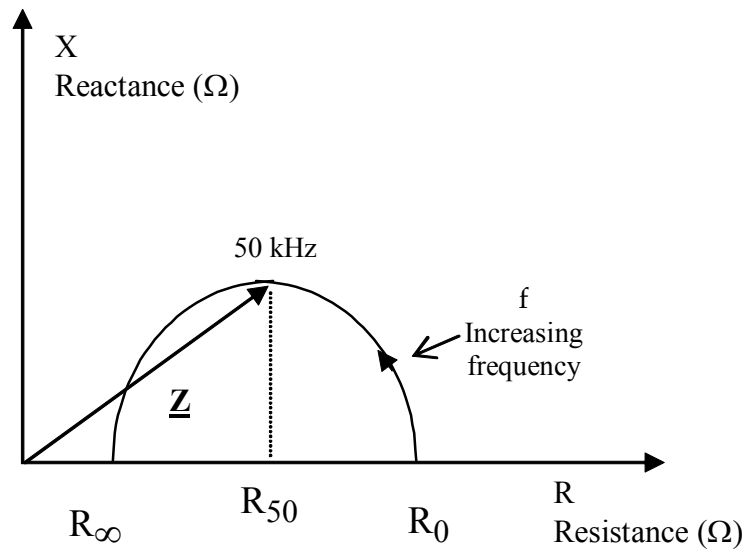

Fig. 5. Cole-Cole model 


\subsubsection{Theories used}

Three techniques are generally described, using multiple frequencies, high and low frequencies, and a unique $50 \mathrm{kHz}$ frequency.

The multifrequency mode is the most widely studied. It is based on the Cole-Cole model and allows differentiation between intra- and extra-cellular volumes. But the relationship between resistance and water volume is not linear, so that the multifrequency mode is associated with Hanaï theory, which takes into account the non-conductive element, connected in a parallel, to distinguish the different compartments. The parallel model described by Fricke takes into account the conductive elements exhibiting a certain degree of resistance connected in parallel and isolated by a cell membrane. This method has been validated by Van Loan $[29,30,31]$. At high and low frequencies, the Hanaï theory takes into account the presence of non-conductive elements connected in parallel. The measurement improvement mainly concerns the extracellular compartment $[32,33]$. At the unique $50 \mathrm{kHz}$ frequency, the theory enables a measurement of TBW (and lean body mass) [28]. Many different formulas have been published, using the term: $H^{2} / R_{50}$, where $H$ is the subject's height in $\mathrm{cm}$ and $R_{50}$ the wrist-ankle resistance. These methods have not been validated and generally cannot detect small volume changes. This limitation may be related to the relative uniformity of tissue elements considered in the formulas which do not take into consideration changes in tissue composition. Simple calculations allow extrapolations for extracellular water [34].

These different theories can then be applied to measure body segments or the whole body $[35,36,37,38]$. For segmental measures, the mathematical models remain empirical and actual measures remain dependent on electrode position. Differences appear in comparison with whole body measures with a trend to underestimate water loss during a dialysis session compared with a segmental measurement, particularly in the event of hypotension [39]. One advantage is that the segmental technique would be less sensitive to changes in patient position from one measure to the next [40].

\subsubsection{From theory to measure}

The problem is then to transform a resistance measurement into a calculation of body composition. Geometry plays an important role. As seen in Figure 6, three objects with a constant height exhibit the same resistance from top to bottom, despite their different volumes [41].
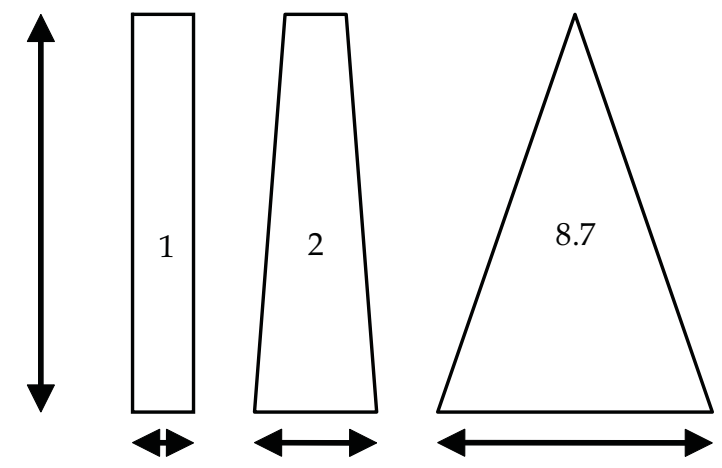

Fig. 6. Complexity of the body geometry-measurement relationship 
Thus in many models, the human body is considered to be the sum of five compartments (Fig. 7) (the four limbs and the trunk), with a dimension homogeneity factor $K_{b}$.

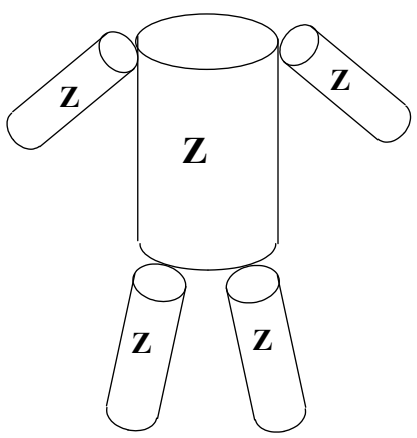

Fig. 7. The 5-compartment human body model

The homogeneity factor is calculated from the resistance of a cylinder $(\mathrm{R})$ which is function of its resistivity $(\rho)$, its length (L) and its cross-section (A) [42]

$$
R=\rho \frac{L}{A}
$$

Then, by calculating the volume of the cylinder, and separating, arms, legs and trunk, the following formula is established with $l$ for leg, $t$ for trunk and $a$ for arm:

$$
R=\rho 4 \pi \cdot\left(\frac{L_{1}}{C_{1}^{2}}+\frac{L_{t}}{C_{t}^{2}}+\frac{L_{a}}{C_{a}^{2}}\right)
$$

This formula, together with the classical formula for the volume of a cylinder, yields the dimension homogeneity constant, $\mathrm{K}_{\mathrm{B}}$, which represents a characteristic anthropometric parameter independent of the electrical parameters.

$$
\begin{gathered}
V=K_{B} \rho \frac{L^{2}}{A} \\
K_{b}=\frac{1}{L^{2}} \cdot\left(\frac{L_{1}}{C_{l}^{2}}+\frac{L_{t}}{C_{t}^{2}}+\frac{L_{a}}{C_{a}^{2}}\right)\left(2 L_{a} C_{a}^{2}+2 L_{1} C_{1}^{2}+L_{t} C_{t}^{2}\right)
\end{gathered}
$$

\subsubsection{Formula validity and limitations}

When these formulas are applied to measure body composition, the result is always significantly different from gold standard measurements. Thus Fenech [43] and Jaffrin [44] proposed a direct calculation of TBW using the same method as for extracellular water, assuming that TBW is a homogeneous quantity of fluid. The mean resistivity of this TBW was validated by comparison with body composition data obtained using the DEXA method. Jaffrin then raised the question of extrapolating total body resistance from the resistance value measured at $50 \mathrm{kHz}$ [45], using a proportional intermediary multiplier. 
Finally, the superiority of multifrequency impedance over the monofrequency impedance has not been demonstrated. Similarly no one formula has been found superior to the others.

\subsection{Available clinical applications}

Several studies have been conducted in hemodialysis patients. Three approaches have been particularly fruitful, the nomovolemia/hypervolemia curves established by Chamney [46, 47, 48, 49], the resistance/reactance curves by Piccoli [50, 51, 52] and Zhu's continuous curves $[53,54]$. Although essential, these approaches will not be detailed here. Readers may usefully refer to the cited references.

\subsubsection{Problems and solutions}

\subsubsection{Changes of body position}

It has been shown that resistance/reactance curves are modified reversibly by changes in body position (reclining, sitting, upright position). Physical studies have demonstrated a modification in the lines of current passing through the body with changes in body position. In addition, length and resistance measures vary [55]. In the healthy subject, comparison of whole body and segmental measures shows an increase in resistance in all sectors (peripheral and central compartments) when moving from the upright to the reclining position [56, 57]. This is logical for the peripheral sector, related to the decrease in water in this sector which is redistributed to the central sector by cancelation of the gravity effect. However, surprisingly, resistance increases in the trunk, despite the increase in water. One explanation is that impedancemetry poorly evaluates the central sector. In the dialysis patient, and using the segmental mode, resistances vary with electrode position. The volumes calculated using the segmental mode are much higher than with the whole body mode and than with the anthropometric formulas [58]. In conclusion, segmental measures are insufficient to eliminate artifacts related to changes in body position.

\subsubsection{Underestimation of extracellular volume variations in dialysis patients}

These calculations are made from variations in plasma sodium content, accepting the hypothesis of zero sodium exchange between intra- and extra-cellular compartments or with the dialysis solute (obviously a false assumption). These results show that the contribution of the extracellular volume is superior to that measured by impedancemetry, with certain aberrant results [59,60]. Conductivity variability is well known in dialysis and is most likely responsible for these problems of fluctuating resistivity for different compartments during dialysis.

\section{Experimental materials and methods}

\subsection{Tanita (TBF-300)}

Tanita (TBF-300) is a device for measuring body impedance between the two legs. It is simple to use and does not require electrodes. Manufactured in Japan, the devise presents as a weight scale with a built-in body composition analyzer which calculates TBW, total body fat and fat-free body mass [61]. The National Institute of Health has not validated the results. For these measurements, the person stands barefoot on a four-point platform. Impedance measurements are made using a high frequency $(50 \mathrm{kHz})$ and low intensity $(500$ $\mu \mathrm{A}$ ) current between the feet. (formula are not known) 


\subsection{Body composition monitor}

The body composition monitor (BCM) was developed by Fresenius ${ }^{\circledR}$ Medical Care. BCM is a rapid non-invasive 4-electrodes method for monitoring body composition by measuring the electrical impedance response to a multifrequency signal ( 50 frequencies from $5 \mathrm{kHz}$ to 1 $\mathrm{MHz}$ ). The Cole-Cole model is applied, with Hanaï mixture theory, to distinguish extracellular water (ECW), intracellular water (ICW) and total body water. Integrated software can also display over-hydration status. The device has been validated in healthy people, in comparison with reference methods (isotopic dilution).

The advantage of this device is to provide a quantitative diagnosis of over-hydration. The model is based on tissue hydration constants observed in the healthy individual which can then be applied to quantify excess water in kidney disease patients. The validation study, based on gold standard methods (bromide dilution for the extracellular compartment, deuterium dilution for TBW, potassium dilution for intracellular compartment and DEXA for fat mass), included 500 healthy subjects. The measures were then compared with the hemodialysis patient, before and after dialysis. This technique assumes that intracellular water, fat-free mass, and fat mass remain unchanged during the course of the dialysis session despite the ultrafiltration [62].

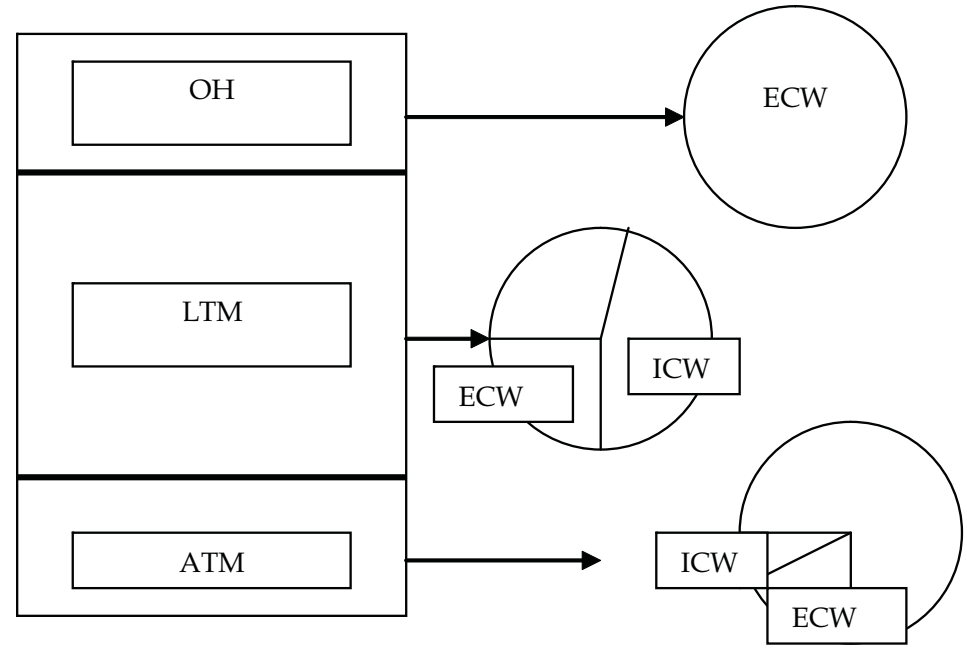

Fig. 8. Physiological model used by the body composition monitor (BCM) to determine the hydration constant of tissues and thus overhydration $(\mathrm{OH})$. ATM: adipocyte tissue mass; LTM: lean tissue mass

The novel aspect of this model is the introduction of a third compartment corresponding to the excess fluid (Fig. 8) or over-hydration $(\mathrm{OH})$ in comparison with the healthy individual. This over-hydration is considered to concern the extracellular compartment exclusively. Lean body mass contains exactly $73.2 \%$ water and includes the intra- and extra-cellular compartments, the intracellular compartment predominating. Lean body mass includes protein (muscles) and mineral (bone) matter. The sum of the intracellular compartment plus the protein mass defines the cell mass. Adipose tissue mass, or fat mass, contains $26.8 \%$ water, the extracellular compartment predominates. The notion of a third compartment avoids the problems related to the fact that the ratio between the extracellular and 
intracellular water is not constant. Comparing total and extracellular water measurements obtained in the healthy population and in the dialysis population defines the notion of overhydration. Time-course curves of the different compartments provide a picture of the evolving state of hydration.

In Figure 9, the notion of hydration status in the dialysis patient is combined with systolic blood pressure. Four sectors are defined. I: over-hydration and hypertension, II: normohydration and hypertension, III: normo-hydration and normo-tension IV: over-hydration and normo- or hypo-tension. Trends can be plotted.

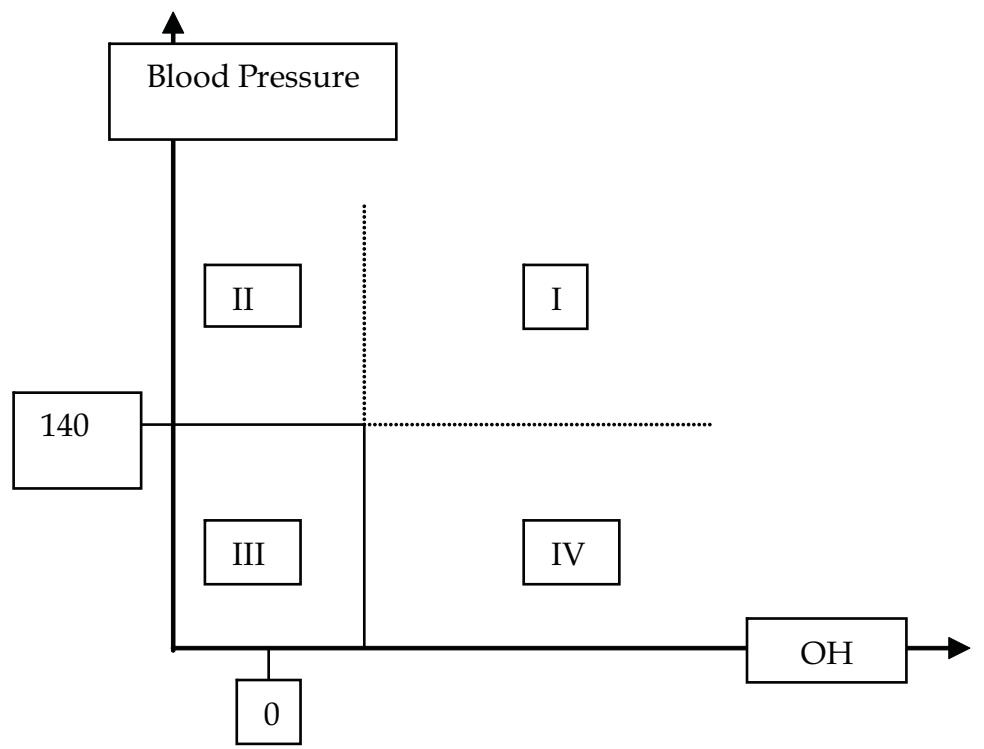

Fig. 9. Hydration reference

\subsection{Experimental works and results 3.3.1 Hemodialysis patients}

\subsubsection{Elaborating a dry-weight index using Tanita}

We first used the Tanita TBF-300 analyzer to obtain bioimpedance measures in 238 hemodialysis sessions. The mean total body water (TBW) was $41.9 \pm 7.9 \mathrm{~kg}$ before sessions and $39.6 \pm 7.3 \mathrm{~kg}$ after sessions. Mean body weight was $76.6 \pm 18.2 \mathrm{~kg}$ before and $74.2 \pm 17.1 \mathrm{~kg}$ after. Statistically, body weight remained stable during this study and was thus valid for assessing reliability. This enabled the definition of patient populations used to hypothesize the pathophysiology of hyperhydration, dehydration and normohydration state based on a body-weight index (Fig.10). Change in weight $(\Delta \mathrm{W})$ and TBW $(\Delta \mathrm{TBW})$ was noted and compared with ultrafiltration. In theory, these three variables should be similar, because during dialysis sessions, the ultrafiltration applied corresponds to a subtraction of water, and consequently to the change in $\mathrm{w}$ or TBW before and after the water depletion. But net ultrafiltration and $\Delta \mathrm{W}$ correlated poorly $(\mathrm{c}=0.62)$ with $\triangle \mathrm{TBW}$. This led to the definition of a dry-weight index, I. It was hypothesized that this Index would be 0 when patients reach their dry weight: $\mathrm{I}=\Delta \mathbf{W}-\Delta \mathrm{TBW}$ 


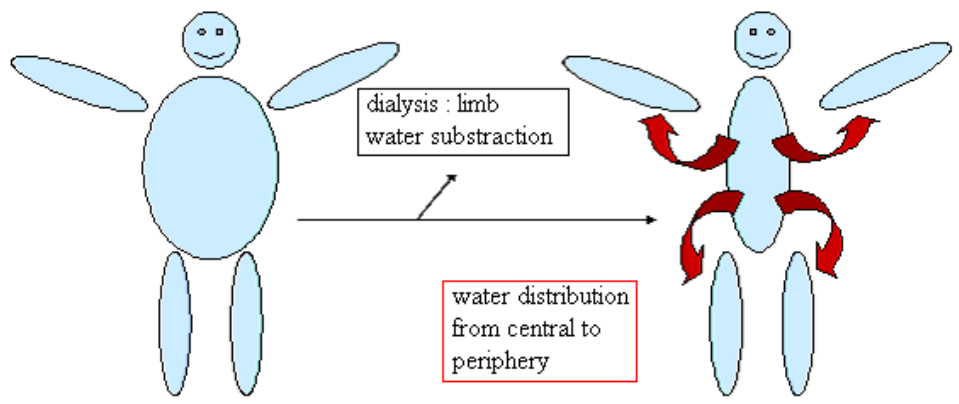

Low TBW variation, so high Index

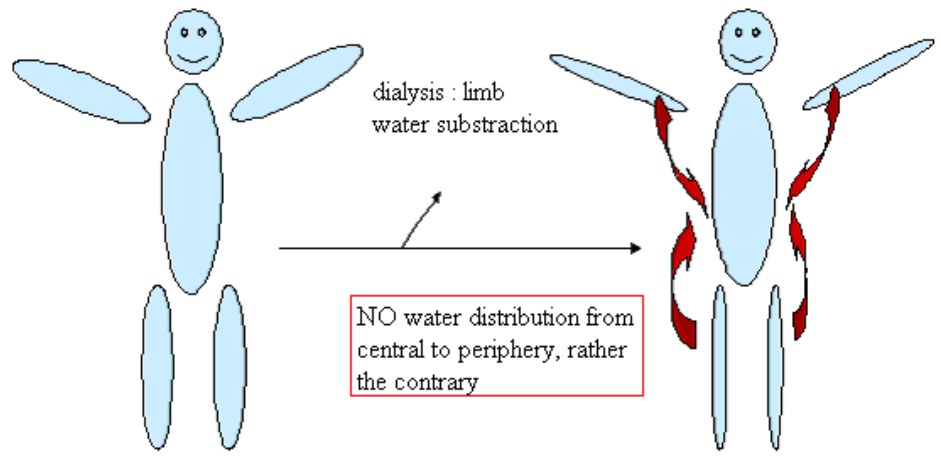

High TBW variations, so low or negative index

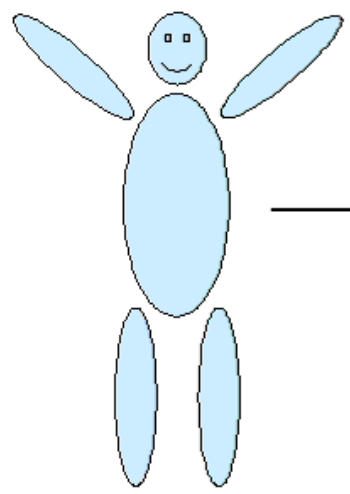

dialysis : limb

water substraction

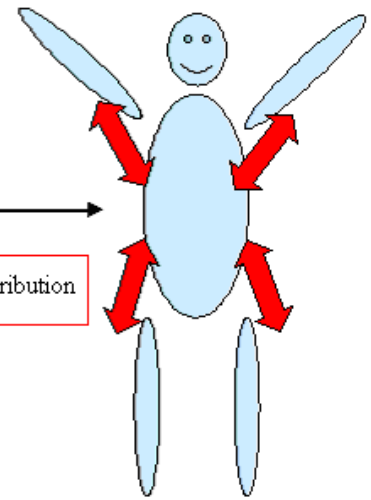

good measure of TBW variations, so a null index

Fig. 10. Pathophysiology of hydration state. upper panel: hyperhydration; middle panel: dehydration; lower panel: normo-hydration. TBW = total body water, $\mathrm{W}=$ body weight. Dry-weight index $: \mathbf{I}=\Delta \mathbf{W}-\Delta \mathrm{TBW}$ 


\subsubsection{Body composition monitoring of the elderly hemodialysis patients}

Body composition was monitored for a 1.5-month period under standardized conditions in 10 hemodialysis patients aged over 80 years with the BCM. The data collected (Table 1) showed that these elderly patients exhibited a different water distribution pattern during ultrafiltration and also different refilling behavior. Thus ultrafiltration did not fully reflect changes in over-hydration. The only valid and clearly coherent measures were those recorded before the dialysis session. While the Cole-Cole model remained valid, implanted devices or specific clinical situations such as heart failure affected the impedance results. Pacemaker behavior (patient $n^{\circ} 8$ ) was unaffected by the very small current used, but the presence of conductive metal (orthopedic prosthesis in patient 10) modified the bioimpedance measures. In such patients, change in weight and ultrafiltration followed a similar pattern, but TBW recordings were obviously incorrect in light of the weight loss (Table 1) and the over-hydration diagnosis (Table 2).

\begin{tabular}{|c|c|c|c|c|c|c|c|c|c|c|}
\cline { 2 - 12 } \multicolumn{1}{c|}{} & \multicolumn{2}{c|}{$\Delta$ Weight (kg) } & \multicolumn{2}{c|}{ UF (1) } & \multicolumn{2}{c|}{$\Delta$ TBW (1) } & \multicolumn{2}{c|}{$\Delta$ ECW (l) } & \multicolumn{2}{c|}{$\Delta$ ICW (l) } \\
\cline { 2 - 12 } \multicolumn{1}{c|}{} & mean & SD & mean & SD & mean & SD & mean & SD & mean & SD \\
\hline patient 1 & 2,03 & 0,36 & 2,1 & 0,59 & 2,5 & 0,96 & 2,07 & 0,47 & 0,47 & 0,47 \\
\hline patient 2 & 1,22 & 0,2 & 1,22 & 0,32 & 1,26 & 0,41 & 1,12 & 0,19 & 0,16 & 0,29 \\
\hline patient 9 & 1,43 & 0,68 & 1,76 & 0,72 & 1,53 & 0,46 & 1,2 & 0,62 & 0,3 & 0,82 \\
\hline patient 3 & 1,58 & 0,3 & 1,3 & 0,23 & 0,78 & 0,43 & 0,55 & 0,06 & 0,23 & 0,46 \\
\hline patient 5 & 1,52 & 0,51 & 1,28 & 0,31 & 0,46 & 1,46 & 0,82 & 0,48 & $-0,3$ & 0,17 \\
\hline patient 6 & 2,9 & 0,29 & 2,8 & 0,15 & 1 & 0,71 & 1,85 & 0,35 & $-0,95$ & 1,06 \\
\hline patient 10 & 1,3 & 0,99 & 1,52 & 0,55 & 0,77 & 0,75 & 0,6 & 0,36 & 0,17 & 0,42 \\
\hline patient 4 & 1,33 & 0,22 & 1,18 & 0,16 & 2,7 & 0 & 1,7 & 0,17 & 0,97 & 0,15 \\
\hline patient 8 & 2,63 & 0,43 & 2,34 & 0,34 & 6,83 & 2,48 & 1,5 & 0,53 & 5,43 & 2,94 \\
\hline patient 7 & 1,53 & 2,19 & 0,72 & 0,19 & 0,78 & 0,57 & 1 & 0,68 & $-0,25$ & 0,17 \\
\hline
\end{tabular}

Table 1. Participation of fluid compartments during ultrafiltration (UF)

Four patient profiles could be identified from the data presented in Table 1.

- Patients 1, 2 and 9 exhibited good correlation between weight loss, applied ultrafiltration, and TBW variation during dialysis sessions.

- Patients 3, 5, 6 and 10 exhibited a different type of behavior. The decline in TBW from the beginning to the end of the dialysis session was underestimated as compared with the variation in weight or applied ultrafiltration.

- Inversely, for patients 4 and 8, the decline in TBW during dialysis was overestimated, compared with the very similar variations in weight or ultrafiltration.

- In one patient $\left(n^{\circ} 7\right)$, ultrafiltration and TBW correlated well with each other, but not with weight loss.

As expected, dialysis-related water depletion mainly involved a reduction of the extracellular compartment compared with the intracellular compartment. This well-known phenomenon is called refilling. In three patients $\left(n^{\circ} 5,6\right.$ and 7$)$, the bioimpedance measures predicted fluid overload in the intracellular compartment, with a smaller fall in TBW than in extracellular water. This might be related to a "reverse refilling" phenomenon. For patient 
$\mathrm{n}^{\circ} 8$ who had a pacemaker, fluid removal seemed to occur exclusively from the intracellular compartment.

\begin{tabular}{|c|c|c|c|c|}
\cline { 2 - 5 } \multicolumn{1}{c|}{} & \multicolumn{2}{c|}{ OH before (1) } & \multicolumn{2}{c|}{ OH after (1) } \\
\cline { 2 - 5 } \multicolumn{1}{c|}{} & mean & SD & mean & SD \\
\hline patient 1 & 0,1 & 0,49 & $-1,77$ & 0,55 \\
\hline patient 2 & 1,7 & 0,21 & 0,72 & 0,13 \\
\hline patient 3 & 0,78 & 0,11 & 0,53 & 0,21 \\
\hline patient 4 & 0,98 & 0,29 & $-0,17$ & 0,12 \\
\hline patient 5 & 0,72 & 0,33 & $-0,1$ & 0,39 \\
\hline patient 6 & 2,6 & 0,71 & 0,57 & 0,15 \\
\hline patient 7 & 0,62 & 0,63 & $-0,15$ & 0,77 \\
\hline patient 8 & 0,5 & 1,59 & 1,63 & 0,8 \\
\hline patient 9 & 0,84 & 0,65 & $-0,2$ & 0,26 \\
\hline patient 10 & $-0,6$ & 0,12 & $-0,97$ & 0,21 \\
\hline
\end{tabular}

Table 2. Body composition monitoring (BCM) diagnosis of over-hydration $(\mathrm{OH})$ status

The over-hydration data presented in Table 2 show that, according to the BCM findings, all patients except two ( $\mathrm{n}^{\circ} 8$ and 10), were over-hydrated at dialysis onset and underhydrated at dialysis end. Comparing the clinical diagnosis of over-hydration established by the nephrologist programming the ultrafiltration at the beginning of the session (Table 1) with the over-hydration status diagnosis established by BCM before dialysis (Table 2) revealed different trends. The nephrologist tended to overestimate over-hydration before dialysis while the BCM tended towards an underestimation. At the end of the ultrafiltration six of the patients were dehydrated, but not far from their dry weight, with a difference of $\leq 0.5$ liters from normo-hydration.

\subsubsection{Body composition monitoring in living-donor transplant recipients}

We prospectively studied five transplanted patients who had received a living-donor graft after a period of hemodialysis. These five patients had exhibited particularly rapid recovery of normal renal function after transplantation. We took bioimpedance measures before surgery and several times during a mean 2-months period after transplantation. Our findings produced a bell-shaped curve showing weight gain with increasing over-hydration followed by weight loss with normo-hydration, in correlation with the normalization of kidney graft function (Table 3).

\begin{tabular}{|c|c|c|}
\hline BCM diagnosis & Day 1 post-transplantation & Day 50 post-transplantation \\
\hline Over-hydration & $1.04 \pm 0.7$ liters & $0.56 \pm 0.3$ liters \\
\hline
\end{tabular}

Table 3. Over-hydration after kidney transplantation

Clinically, the patients' blood pressure improved and edema disappeared. For example, one patient weighed $48.4 \mathrm{~kg}$ on day 4 post-transplantation and $47.3 \mathrm{~kg}$ on day 10. There was a reduction of the hyperhydration, the Cole-Cole curves shifting to the right (Fig. 11), and an increase in maximal reactance $(X)$ (from 50.6 to $62.5 \mathrm{Ohm}$ ). The frequency at which this reactance reached its maximum also declined from $60 \mathrm{kHz}$ to $50 \mathrm{kHz}$, a frequency which is commonly observed in normo-hydration subjects. 
$\mathrm{X}(\mathrm{Ohm})$

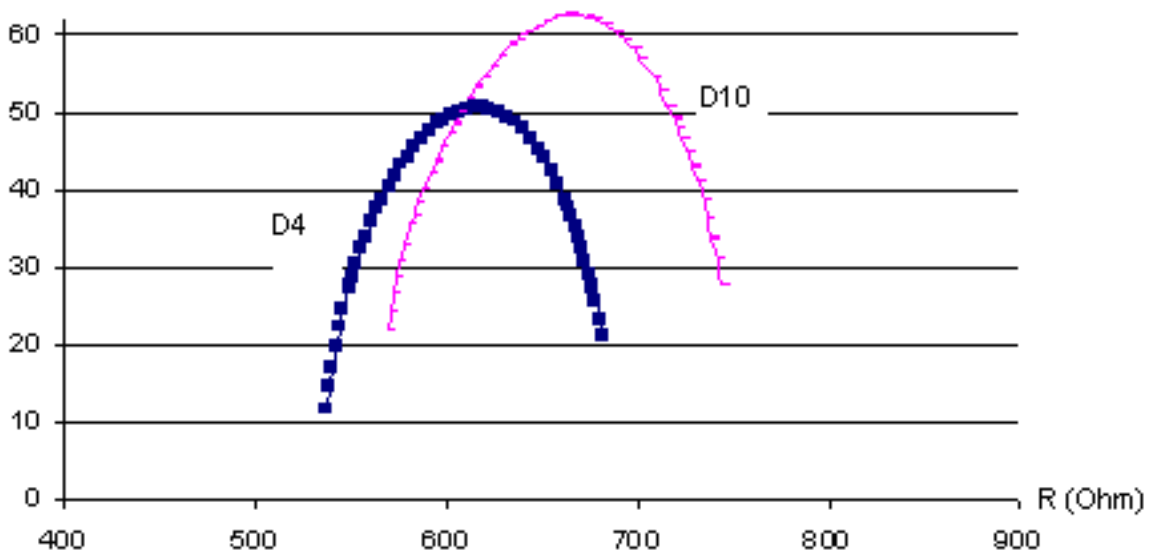

Fig. 11. Cole-Cole time curves after kidney transplantation. In blue at Day 4 post transplantation $(\mathrm{OH}+0.5)$, in red at Day 10 post transplantation $(\mathrm{OH}+0.6)$

\subsubsection{Body composition monitoring in acute kidney failure}

We studied three patients with acute reversible kidney disease. Body composition monitoring was started at the time of the acute disease and continued to the recovery period. Results (impedance, reactance, phase shift, Cole-Cole curve) showed an overhydration period that disappeared with the normalization of kidney function. The time course of improved hydration status in this patient is represented in Figure 12. Overhydration and weight variations followed the same pattern, rising and declining in parallel.

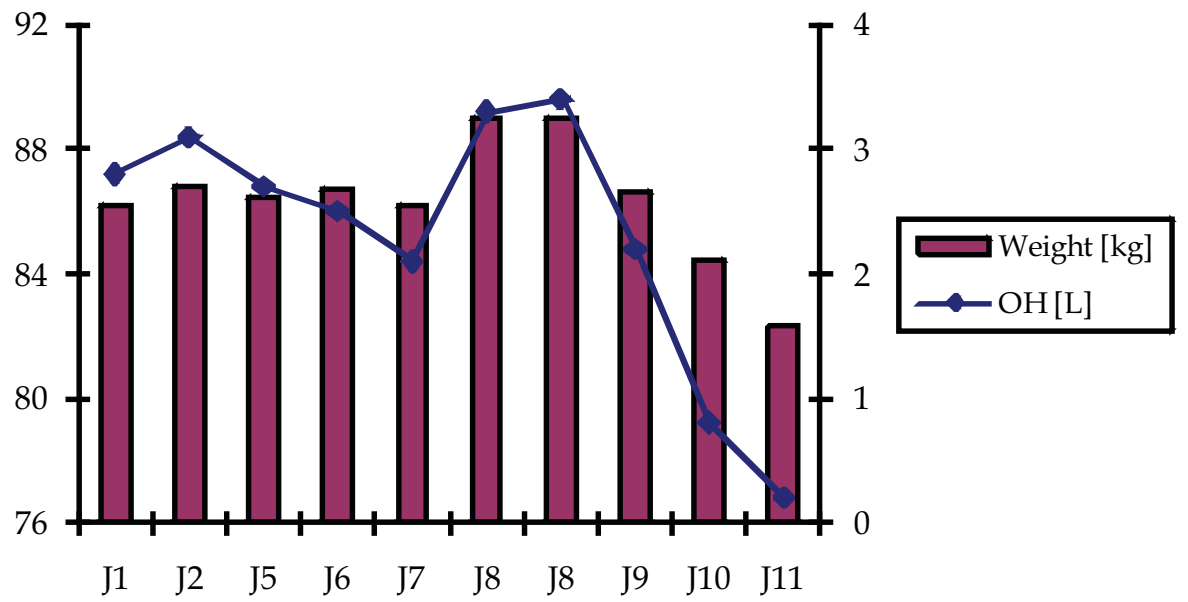

Fig. 12. Hydration status time course in a patient recovering normal kidney function. 


\begin{tabular}{|l|c|c|}
\cline { 2 - 3 } \multicolumn{1}{c|}{} & Day 1 & Day 11 \\
\hline weight $(\mathrm{kg})$ & 86.2 & 82.3 \\
\hline Over-hydration (Kg) & 2.8 & 0.2 \\
\hline Maximal reactance & 25 & 37.7 \\
\hline Resistance $(\mathrm{Ohm})$ & 410 & 540 \\
\hline Frequency $(\mathrm{kHz})$ & 67 & $5 \mathrm{O}$ \\
\hline
\end{tabular}

Table 4. Resistance and frequency values at maximal reactance

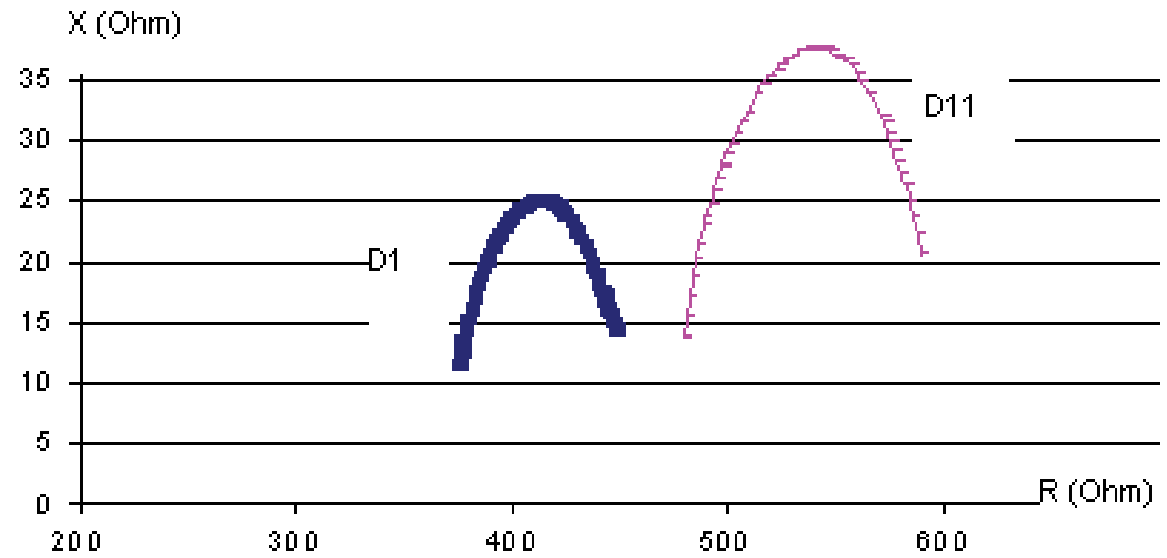

Fig. 13. Cole-Cole curves with recovery of kidney function from Day 1 (blue) to Day 11 (red)

Figure 13 also shows that the Cole-Cole curves shifted to the right as hydration status returned to normal. This right shift being the expression of lesser hyperhydration. On day 1 , the resistance levels at 0 and $\infty$ frequency went from 450 to $370 \mathrm{Ohms}$ with a maximum reactance of 26 for a resistance of 410 . By day 11, the curves had shifted to the right, corresponding to lesser hyperhydration, weight loss, and a diagnosis of lower overhydration. These values ranged from 590 to $480 \mathrm{Ohms}$ and maximal reactance of 37.7 with a corresponding resistance at 540 (Table 4). These physical elements also corresponded to clinical reality since blood pressure levels returned to normal with involution of lower limbs edema.

\section{Conclusion}

Bioimpedance is an easy-to-use tool providing a useful evaluation of patients' dry weight. The method remains limited due to the lack of standards of normality. Moreover, changes in electrolytes, red cells, protein, body temperature or implanted devices affect bioimpedance measures, further compromising use of bioimpedance for determining the dry weight or normal hydration level of hemodialysis patients. Nevertheless, measurable values such as impedance, reactance, or phase shift enable the identification of patient groups and the description of trends. Impedance, reactance and phase shift can be used to identify population groups with similar behavior patterns useful for determining dry weight or detecting hydration disorders. Analysis of correlations between physical data and clinical findings is another point of interest discussed in this chapter. 


\section{References}

[1] Charra, B. (1998). "Dry weight in dialysis": the history of a concept. Nephrol Dial Transplant, Vol 7, pp.1882-1885

[2] Wabel, P.; Moissl, U.; Chamney, P.; Jirka, T.; Machek, P.; Ponce, P. \& Wizemann, V. (2008). Towards improved cardiovascular management: the necessity of combining blood pressure and fluid overload. Nephrol Dial Transplant, Vol 23, pp.2965-2971

[3] Kooman, J.; Van des Sande, F. \& Leunissen, K. (2009). Wet or dry in dialysis-Can new technologies help. Seminars in Dialysis, Vol 22, No.1, (January 2009), pp.9-12, Maastricht, The Netherlands

[4] McGee, S.; Abernethy, Wb. \& Simel, DL. (1999). The rational clinical examination. Is this patient hypovolemic? JAMA, Vol 281, pp.1022-1029

[5] Ishibe, S.; Peixoto, A. (2004). Methods of assessment of volume status and intercompartmental fluid shifts in hemodialysis patients: implications in clinical practise. Seminars in Dialysis, Vol 17, No.1, (January 2004), pp.37-43

[6] Kraemer, M. (2006). A new model for the determination of fluid status and body composition from bioimpedance measurements. Physiol Measurement, Vol 27, pp.901-919

[7] Jaffrin, M.Y, Fenech, M., de Fremont, J.F. \& Tolani, M. (2002). Continuous monitoring of plasma, interstitial and intracellular fluid volumes in dialyzed patients by bioimpedance and hematocrit measurements. ASAIO J, Vol, 48, pp.326-333

[8] Morucci, JP.; Chauveau, N. ; Rigaud, B. ; Felice, C.\& Marcili, P. (1996). Bioelectrical impedance techniques in medicine. Crit Rev Biomed Eng, Vol 24, pp.257-677

[9] Cole, K. (1928). Electrical impedance of suspension of spheres. J Gen Physiol, Vol 12, pp. 29-36

[10] Cole, K. (1934). Alternating current conductance and direct current excitation of nerve. Science, Vol 79, pp.164-165

[11] Cole, K. (1940). Permeability and impermeability of cell membranes for ions. Cold Spring Harbor Symp. Quant. Biol Vol 8, pp. 110-122

[12] Foster, K.R. \& Schwan, H.P. (1994). Dielectric properties of tissues. In C Polk, E Postow (eds), Handbook of Biological Effects of Electromagnetic Fields, $2 \mathrm{~d}$ ed

[13] Fricke, H. (1925). A mathematical treatment of the electrical conductivity and capacity of disperse system. II. The capacity of a suspension of conducting speroids surrounded by a nonconducting membrane for a current of low frequency. Phys Rev, Vol 26, pp. 678-681

[14] Ho, LT.; Kushner, RF.; Schoeller, DA.; Gudivaka, R. \& Spiegel, DM. (1994). Bioimpedance analysis of total body water in hemodialysis patients. Kidney, Vol 46, pp.1438-1442

[15] Zhu, F.; Schneditz, D. \& Levin NW. (1999). Sum of segmental bioimpedance analysis during ultrafiltration and hemodialysis reduces sensitivity to changes in body position. Kidney Int, Vol 56, pp.692-699

[16] Zhu, F.; Schneditz, D.; Wang, E.; Martin, K.; Morris, AT. \& Levin, NW. (1998).Validation of changes in extracellular volume measured during hemodialysis using a segmental bioimpedance technique. ASAIO J, Vol 44, pp541-545

[17] Chanchairujira, T. \& Mehta, RL.( 2001) Assessing fluid change in hemodialysis: whole body versus sum of segmental bioimpedance spectroscopy. Kidney Int, Vol 60, pp.2337-2342 
[18] Gudivaka, R.; Schoeller, D.; Kushner, R. \& Bolt, M. (1999). Single- and multifrequency models for bioelectrical impedance analysis of body water compartments. J Appl Physiol, Vol 87, pp.1087-1096

[19] Chertow, G.; Lazarus, J.; Lew, N.; Ma, L. \& Lowrie E. (1997). Bioimpedance norms for the hemodialysis population. Kidney Int, Vol 52, pp.1617-1621

[20] Buchholz, A.; Bartok, C. \& Schoeller, D. (2004). The validity of bioelectrical impedance models in clinical populations. Nutr Clin Pract, Vol 19, p.433-446

[21] Shulman, T., Heidenheim, A., Kianfar, C., Shulman, S. \& Lindsay, R. (2001). Preserving central blood volume changes in body fluid compartments during hemodialysis. ASAIO J, Vol 47, pp. 615-618

[22] Lafargue, L.A., Cabrales, L.B. \& Larramendi, R.M. (2002). Bioelectrical parameters of the whole human body obtained through bioelectrical impedance analysis. Bioeelectromagnetics, Vol 23, pp.450-454

[23] Aloia, J.F., Vaswabi, A., Flaster, E. \& Ma, R. (1998). Relationship of body water compartments to age, race, and fat-free mass. J Lab Clin Med, Vol 132, pp.483-490

[24] Basile, C., Vernaglione, L., Di Lorio, B., Bellizzi, V., Chimienti, D., Lomonte, C., Rubino, A. \& D'Ambrosino, N. (2007). Development and validation of bioimpedance analysis prediction equation for dry weight in hemodialysis patients. Clin J Am Soc Nephrol, Vol 2, pp.675-680

[25] Lukaski, H.C. (1996). Biological indexes considered in the derivation of the bioelectrical impednce analysis. Am J Clin Nutr, Vol 64, pp.397-404

[26] Kushner, R.F. \& Schoeller, D.A. (1986). Estimation of total body water by bioelectrical impedance analysis. Am J Clin Nutr, Vol 44, pp.417-424

[27] Thomasset, A. (1963). Bio-electrical properties of tissue impedance measurements. Lyon Med, Vol 209, pp.1325-1352

[28] Hoffer, E.C., Meador, C.K. \& Simpson, D.C. (1969). Correlation of whole-body impedance with total body water volume. J Appl Physiol, Vol 27, pp.531-534

[29] Van Loan, M.D., Withers, P., Matthie, J. \& Mayclin, P.L. (1993). Use of bioimpedance spectroscopy to determine extracellular fluid (ECF), intracellular fluid (ICF), total body water (TBW), and fat free mass (FFM). In: human body composition: in vivo methods, models and assessment, edited by Ellis, K.J. \& Eastman, J.D., pp.67-70, New York: Plenum

[30] Van Marken Lichtenbelt, W.D., Westerterp, K.R., Wouters, L. \& Luijendijk, S. (1994). Validation of bioelectrical impedance measurements as a method to estimate bodywater compartments. Am J Clin Nutr, Vol 60, pp.159-166

[31] Ho, L.T., Kushner, R., Schoeller, A., Gudivaka, R. \& Spiegel, D.M. (1994). Bioimpedance analysis of total body water in hemodialysis patients. Kidney Int, Vol 46, pp.14381442

[32] Deurenberg, P., Andreoli, A. \& De Lorenzo, A. (1996). Multifrequency bioelectrical impedance: a comparison between the Cole-Cole modeling and Hanaï equations with the classical impedance index approach. Ann Hum Biol, Vol 6, pp.31-40

[33] Segal, K.R., Burastero, S., Chun, A., Coronel, P., Pierson, R.N. Jr, \& Wang, J. (1991). Estimation of extracellular and total body water by multiple-frequency bioelectrical -impedance measurement. Am J Clin nutr, Vol 54, pp.26-29 
[34] Lukaski, H.C. \& Bolonchuk, W.W. (1988). Estimation of body fluid volumes using tetrapolar bioelectrical impedance measurements. Aviat Space Environ Med, Vol 59, pp.1163-1169

[35] Park, J. Yang, W.S., Kim, S.B., Paark, S.K., Lee, S.K., Park, J.S. \& Chang, J.W. (2008). Usefulness of segmental bioimpedance ration to determine dry body weight in new hemodialysis patients: a pilot study. Am J of Nephrol, Vol 29, pp.25-30

[36] Levin, N.W., Zhu, F., Seibert, E., Ronco, C. \& Kuhlmann, M.K. (2005). Use of segmental multifrequency spectroscopy in hemeodialysis. Contrib to Nephrol, Vol 149, pp.162-167

[37] Chanchairujira, T. \& Mehta, R.L. (2001). Assessing fluid change in hemodialysis : whole body versus sum of segmental bioimpedance spectroscopy. Kidney Int, Vol 60, pp.2337-2342

[38] Zhu, F., Ronco, C., Schneditz, D., De Simone, L. \& Levin, N.W. (2000). Estimation of dry body weight by segmental bioimpedance analysis during hemodialysis. ASAIO J; Vol 46, pp.221

[39] Zaluska, W.T., Schneditz, D. \& Kaufman, A.M. (1998). Relative underestimation of fluid removal during hemodialysis hypotension measured by whole body bioimpedance. ASAIO J, Vol 44, pp.823-827

[40] Zhu, F., Schneditz, D. \& Levin, N.W. (1999). Sum of segmental bioimpednce analysis during ultrafiltration and hemodialysis reduces sensitivity to changes in body position. Kidney Int, Vol 56, pp.692-699

[41] Foster, K.R. \& Lukeaski, H.C. (1996). Whole-body impedance-what does it measure? Am J Clin Nutr, Vol 64, pp.388-396

[42] De Lorenzo, A., Andreoli, A., Matthie, J. \& Withers, P. (1997). Predicting body cell mass with bioimpedance by using theoretical methods: a technological review. J Appl Physiol, Vol 82, pp.1542-1558

[43] Jaffrin, M.Y. \& Morel, H. (2008). Body fluid volumes measurements by impedance: a review of bioimpedance spectroscopy (BIS) and bioimpedance analysis (BIA) methods. Med Eng Phys, doi: 10.1016/j.medengphy.2008.06.009

[44] Jaffrin, M.Y., Fenech, M., Moreno, M.V. \& Kieffer, R. (2006). Total body water measurement by a modification of the bioimpedance spectroscopy method. Med Biol Eng Comput, Vol 44, PP.873-882

[45] Morel, H. \& Jaffrin, M.Y. (2008). A bridge from bioimpedance spectroscopy to $50 \mathrm{kHz}$ bioimpedance analysis: application to total body water measurements. Physiol Meas, Vol 29, pp.465-478

[46] Matthie, J.R. (2005). Second generaation mixture theory equation for estimating intracellular water using bioimpedance spectroscopy. J Appl Physiol, Vol 99, 780781

[47] Matthie, J.R., Zaroutz, B. \&De Lorenzo, A. (1998). Analytic assessment of various bioimpedance methods used to estimate body water. J Appl Physiol, Vol 84, PP.1801-1816

[48] Moissl, U.M., Wabel, P., Chamney, P.W., Bosaeus, I. \& Levin, N.W. (2006). Body fluid volume determination via body composition spectroscopy in health and disease. Physiol Meas, Vol 27, pp.921-933 
[49] Chamney, P.W., Krämer, M, Rode, C., Kleinekofort, W. \& Wizemann, V. (2002). A new technique for establisching dry weight in hemodialysis patients via whole body bioimpedance. Kidney Int, Vol 61, pp.2250-2258

[50] Piccoli, A. (1998). Identification of opérational clues to dry weight prescription in hemodialysis using bioimpedance vector analysis. Kidney Int, Vol 53, pp.1036-1043

[51] Epstein, B.R. \& Foster K.R. (1983). Anisotropy in the dielectric properties of skeletal muscle. Med Biol Eng Comp, Vol 21, pp.51-55

[52] Piccoli, A., Pastori, G. \& Guizzo, M. (2005). Equivalence of information from single versus multiple frequency bioimpedance vector analysis in hemodialysis. Kidney Int, Vol 67, pp.301-313

[53] Zhu, F., Kuhlmann, M.K. \& Sarkars, S. (2004). Adjustment of dry weight in hemodialysis patients using intradialytic continuous multifrequency bioimpedance of the calf. Int J Artif Organs, Vol 27, pp.104-109

[54] Zhu, F., Leonard, E.F. \& Levin, N.W. (2005). Body composition modeling in the calf using an equivalent circuit model of multi-frequency bioimpedance analysis. Physiol Meas, Vol 26, pp.133-143

[55] Zhu, F.; Schneditz, D., Wang, E. \& Levin NW. (1998). Dynamics of segmental extracellular volumes during changes in body position by bioimpedance analysis. J Appl Physiol, Vol 85, pp.497-504

[56] Zhu, F., Kuhlmann, M.K., Kaysen, G.A., Sarkar, S., Kaitwatcharachai, C., Khilnani, R., Stevens, L., Leonard, E.F., Wang, J., Heymsfield, S. \& Levin N.W. (2006). Segmentspecific resistivity improves body fluid volume estimates from bioimpedance spectroscopy in hemodialysis patients. J Appl Physiol, Vol 100, pp.717-724

[57] Scharfetter, H., Monif, M., Laszlo, Z., Lambauer, T., Hutten, H. \& Hinghofer-Szalkay, H. (1997). Effect of postural changes on the reliability of volumes estimations from bioimpedance spectroscopy data. Kidney Int, Vol 51, pp.1078-1087

[58] Chertow, G.M., Lazarus, J.M., Lew, N.L., Ma, L. \& Lowrir E.G. (1997). Bioimpedance norms for the hemodialysis population. Kidney Int, Vol 52, pp.1617-1621

[59] Kyle, U.G., Bosaeus, I, De Lorenzo, A;, Deurenberg, P., Elia, M;, Gomez, J.F., Heitmann, B.L., Kent-Smith, L., Malchior, J.C., Pirlich, M., Scharfetter, H., Schols, A. \& Pichard, C. (2004). Bioelectrical impedance analysis-part I: review of principles and methods. Clin Nutr, Vol 23, pp.1226-1243

[60] Kyle, U.G., Bosaeus, I, De Lorenzo, A;, Deurenberg, P., Elia, M;, Gomez, J.F., Heitmann, B.L., Kent-Smith, L., Malchior, J.C., Pirlich, M., Scharfetter, H., Schols, A. \& Pichard, C. (2004). Bioelectrical impedance analysis-part II: utilization in clinical practise. Clin Nutr, Vol 23, pp.1430-1453

[61] Jebb, S.A., Cole, T.J., Doman, D., Murgatroyd, P.R. \& Prentice, A.M. (2000). Evaluation of the novel Tanita body-fat analyser to measure body composition by comparision with a four-compartment model. British J of Nutr, Vol 83, pp.115-122

[62] Chamney P.W., Wabel, P., Moissl, U.M., Müller, M.J., Bosy-Westphal, A., Korth, O. \& Fuller, N.J. (2007). A whole-body model to distinguish excess fluid from the hydration of major body tissues. Am J Clin Nutr, Vol 85, pp.80-89 


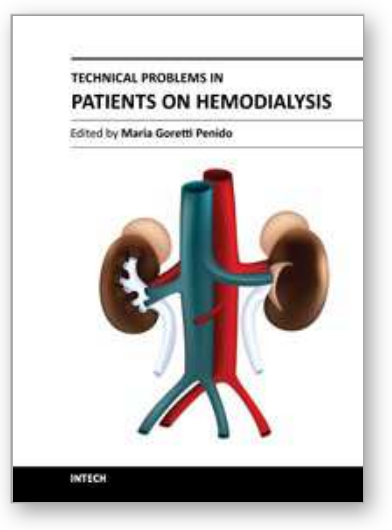

\author{
Technical Problems in Patients on Hemodialysis \\ Edited by Prof. Maria Goretti Penido
}

ISBN 978-953-307-403-0

Hard cover, 312 pages

Publisher InTech

Published online 07, December, 2011

Published in print edition December, 2011

This book provides an overview of technical aspects in treatment of hemodialysis patients. Authors have contributed their most interesting findings in dealing with hemodialysis from the aspect of the tools and techniques used.Each chapter has been thoroughly revised and updated so the readers are acquainted with the latest data and observations in the area, where several aspects are to be considered. The book is comprehensive and not limited to a partial discussion of hemodialysis. To accomplish this we are pleased to have been able to summarize state of the art knowledge in each chapter of the book.

\title{
How to reference
}

In order to correctly reference this scholarly work, feel free to copy and paste the following:

Joëlle Cridlig, Mustapha Nadi and Michèle Kessler (2011). Bioimpedance Measurement in the Kidney Disease Patient, Technical Problems in Patients on Hemodialysis, Prof. Maria Goretti Penido (Ed.), ISBN: 978-953-307403-0, InTech, Available from: http://www.intechopen.com/books/technical-problems-in-patients-onhemodialysis/bioimpedance-measurement-in-the-kidney-disease-patient

\section{INTECH}

open science | open minds

\section{InTech Europe}

University Campus STeP Ri

Slavka Krautzeka 83/A

51000 Rijeka, Croatia

Phone: +385 (51) 770447

Fax: +385 (51) 686166

www.intechopen.com

\section{InTech China}

Unit 405, Office Block, Hotel Equatorial Shanghai

No.65, Yan An Road (West), Shanghai, 200040, China

中国上海市延安西路 65 号上海国际贵都大饭店办公楼 405 单元

Phone: +86-21-62489820

Fax: +86-21-62489821 
(C) 2011 The Author(s). Licensee IntechOpen. This is an open access article distributed under the terms of the Creative Commons Attribution 3.0 License, which permits unrestricted use, distribution, and reproduction in any medium, provided the original work is properly cited. 